\title{
INVESTIGACIONES
}

\section{Haciendo neoliberalismo sobre la marcha. Creación y resignificación de ideas educativas en la dictadura chilena*}

\author{
Doing neoliberalism on the go. \\ Creation and resignification of educational ideas in the Chilean dictatorship
}

\author{
Juan Pablo Venables B. ${ }^{a}$, Claudia Chamorro L. ${ }^{b}$ \\ ${ }^{a}$ Instituto de Historia y Ciencias Sociales. Universidad Austral de Chile. \\ jpvenables@uach.cl \\ ${ }^{b}$ Departamento Antropología. Universidad Alberto Hurtado / Área Museografía. \\ Museo de la Memoria y los DDHH. clauch81@gmail.com
}

\begin{abstract}
RESUMEN
Este artículo propone una reconstrucción jurídica, política e histórica de la implementación de políticas escolares durante la dictadura civil-militar (1973-1990), construyendo un esquema de organización por etapas que complejiza las divisiones expuestas por otros análisis del período. Se rebate la idea que antes de 1979 dominó la inactividad y la confusión, demostrándose que tempranamente se delinearon los principales componentes neoliberales. Para esto, se examinan fuentes legales y documentos deliberativos como las actas de la Junta de Gobierno, prensa, y entrevistas a especialistas y actores clave del período. Se propone una lectura procesual que evidencia su carácter creativo, contingente y cargado de disputas, pues más que una importación mecánica de ideas neoliberales, la dictadura llevó adelante una labor de resignificación y creación eidética. Pensamos que esta lectura favorece la comprensión de la lógica de mercado que opera hoy en el sistema escolar y sus posibilidades de transformación.
\end{abstract}

Palabras claves: Políticas escolares, neoliberalización, lógicas de mercado, Chile.

\begin{abstract}
This paper proposes a legal, political and historical reconstruction of the implementation of school policies during the civil-military dictatorship (1973-1990), building a staged organization scheme that complexes the divisions exposed by other analyzes of the period. We reject the idea that before 1979 inactivity and confusion dominated, demonstrating that the main neoliberal components were early delineated. For this, we examine legal sources and deliberative documents such as the "actas" of the "Junta de Gobierno", press, and interviews with specialists and key actors of the period. We propose a procedural reading that evidences its creative, contingent and full of disputes character. More than a mechanical import of neoliberal ideas, the dictatorship carried out a work of resignification and eidetic creation. We believe that this reading favors the understanding of the market logic that operates in the school system nowadays and its possibilities of transformation.
\end{abstract}

Key words: School policies, neoliberalization, market logics, Chile.

\footnotetext{
* Este artículo fue apoyado por la Vicerrectoría de Investigación, Desarrollo y Creación Artística de la Universidad Austral de Chile, bajo el proyecto DID S-2018-26.
} 


\section{ANTECEDENTES}

La visión que ha predominado en los análisis de las reformas escolares en el período dictatorial propone dos etapas. La primera, conceptualizada como reactiva, ubicada entre 1973 y 1979, estaría marcada por la represión a través de la Doctrina de Seguridad Nacional y la destrucción del modelo dominante basado en el Estado Docente. Así entendida, esta etapa no sugiere la configuración de un nuevo paradigma educativo, más bien, dejaría en evidencia la ausencia de una ruta establecida y una suerte de desorientación en el ámbito educacional (Cox, 1989; PIIE, 1991; Falabella, 2015; Slachevsky, 2015). La segunda etapa ubicada entre 1979 y 1990, sería aquella en la que se define y ejecuta el rumbo neoliberal para el sistema escolar, centrada en dos procesos claves: 1) desconcentración/descentralización financiera, administrativa y pedagógica en los municipios; 2) privatización del sistema en su conjunto mediante la desmejora sistemática de la educación pública y la promoción constante de la educación particular subvencionada (PIIE, 1991; Castro-Paredes, 2012; Slachevsky, 2015; Cox, 1989).

Sin perjuicio de la utilidad de dichas propuestas de periodización, esta investigación arroja otros resultados. La evidencia empírica da cuenta de una lenta «transformación subterránea» de la educación (González, 2015), que comienza desde los primeros meses de la dictadura. Aun cuando sus manifestaciones más nítidas se produzcan desde 1979 en adelante, las directrices ideológicas y las bases jurídicas del sistema comienzan a trabajarse tempranamente desde 1974. En este período "se alistan los mecanismos, se afinan las prácticas y se acumulan las fuerzas para el cambio de institucionalidad que se consolidará más tarde" (González, 2015, p. 34).

En efecto, la revisión de bibliografía, del aparataje legal de las políticas, las actas de la Junta de Gobierno, material de prensa, y entrevistas a especialistas y actores clave del período, indica que el rumbo en materia educativa se fija bastante antes. Nos interesa mostrar empíricamente cómo la toma de decisiones en el gobierno de facto estuvo atravesada por conflictos que expresan la existencia de distintas posiciones al interior de la dictadura. Para relevar estas posiciones, algunos autores se refieren a dos «almas» o «principios» (PIIE, 1991; Slachevsky, 2015; Pérez-Navarro y Rojas-Murphy, 2019), o distintas «tendencias» (Espinoza y González, 1993), «posiciones» (Brunner, 1982) o «voces» (Cox, 1989).

Cox (1989) plantea tres «voces» dentro del debate macro educativo de la dictadura, que estarían en un constante juego de fuerzas: la «voz educativa» (Ministerio de Educación), la «voz económica» (Ministerio de Hacienda y ODEPLAN) y la «voz política» (Augusto Pinochet, pero representada por el Ministerio del Interior). La voz política sería la mediadora entre la educativa y la económica, decidiendo cuál de las dos tiene más peso en determinadas circunstancias. Así, "el esquema muestra con claridad que los actores pueden cambiar de posición, pero no las voces, y que la potencia de una u otra voz [...] no depende tanto de los actores como del 'carácter de la coyuntura' y la decisión de la 'voz política' de dar autoridad a una u otra” (Cox, 1989, p. 16). Como se verá, con excepción de breves períodos en que Vial (1979) y Madariaga (1983) estuvieron a la cabeza del Ministerio de Educación, la «voz educativa» siempre estuvo subordinada a la económica.

Buscamos destacar estos conflictos internos y la manera cómo van resolviéndose en favor de ciertas posiciones, con el fin de mostrar empíricamente que se trató de un proceso creativo, donde se implementa un sistema novedoso, no sencillamente copiado ni definido a priori. En síntesis, se propone un total de siete etapas para comprender el proceso de 
transformación del sistema escolar durante la dictadura. Se abordan con detención las tres primeras (1973-1975; 1976-1978; 1979), con el fin de mostrar que lejos de la desorientación ideológica y puramente reactiva, se trata de una fase determinante para la formulación de una política escolar que aún mantiene vigente varios de sus principios. Las cuatro etapas siguientes van de 1980 a 1990, y dan cuenta de procesos de ejecución de reformas (19801982), crisis (1983-1985), rearticulación (1986), e intento por generar hegemonía (19871990). En todas ellas, se hace un seguimiento a cinco componentes clave del proceso de neoliberalización escolar en Chile: voucher, municipalización, copago, privatización y precarización laboral docente.

\section{PRIMERA ETAPA (1973-1975): CONTROL Y ANTIESTATISMO}

El sello de esta primera etapa que va de 1973 a 1975, es el carácter reactivo hacia las reformas estado-docentistas que venía desarrollando el país durante los últimos cincuenta años y que fueron profundizadas en los gobiernos de Eduardo Frei Montalva (1964-1970) y Salvador Allende (1970-1973). Si bien el foco de preocupación estaba puesto en la Doctrina de Seguridad Nacional y la educación no fue un tema prioritario para la Junta de Gobierno -fue abordado en pocas sesiones y el desarrollo de normativas es escaso-, de igual forma comienzan a vislumbrarse las primeras discusiones y aproximaciones a una lógica privatizadora.

Durante 1973 la educación escolar se trató en cuatro sesiones de la Junta, destacándose dos aspectos. Primero, que el 16 de septiembre, solo a cinco días del Golpe, se planteó como primer punto en tabla que el Ministerio de Educación Pública es "uno de los más conflictivos" (Acta N³, 1973, p. 1). Segundo, el temprano interés de la Junta por encontrar una Política Nacional de Educación. De hecho, el 21 de noviembre de 1973, se crea una comisión para evaluar el estado de desarrollo de la educación nacional. Dicha comisión tuvo como objetivos: "revisar todos los elementos específicos del currículum manipulados discrecionalmente por el Gobierno Marxista y evaluar el proceso de la reforma educacional en curso desde 1965" (Espinoza y González, 1993, pp. 57-58). La comisión contó con la participación de la Federación de Instituciones de Educación Particular (FIDE) (Espinoza y González, 1993), cuyo origen católico muestra la temprana vinculación del Régimen con la Iglesia en materia escolar, y el prematuro interés por incentivar la educación particular.

En 1974 se comienza a delinear de manera más clara la tendencia a favorecer la educación privada y la subsecuente desmejora de la educación pública. En sesión del 10 de enero de 1974, el ministro de Hacienda Contraalmirante Lorenzo Gotuzzo, solicitó a la Junta que "se proporcione subvención a todos los colegios, porque, en caso contrario, el próximo año la educación fiscal se vería muy recargada debido al alto costo de la educación privada" (Acta $N^{\circ} 64,1974$, p. 6). Pese a que la respuesta de la Junta es negativa, ese mismo año se establece el Decreto Ley (DL) 456 para estimular la creación de escuelas particulares (González, 2015). Meses después, en sesión de la Junta de noviembre de 1974, el ministro de Educación Contraalmirante Hugo Castro Jiménez expuso cálculos detallados sobre el menor gasto que implicaría la educación particular subvencionada en relación con la educación pública: "al fisco le sale más rentable mantener esa educación particular subvencionada y no tratar de disminuirla, porque a medida que esta educación particular subvencionada se incremente, se mejore, el fisco puede ir rebajando sus costos" (Acta $\mathrm{N}^{\circ} 172 \mathrm{a}, 1974$, p. 9). 
Las pocas normativas de esta etapa se vinculan con la necesidad de control sobre los distintos actores del espacio educacional y el detrimento de sus atribuciones ${ }^{1}$. Aunque también existen normativas que dan cuenta del prematuro interés por la privatización. En mayo de 1974, con el impulso de la Oficina de Planificación Nacional (ODEPLAN), se promulga el Decreto 456 que deroga la Ley de subvenciones existente desde 1951, señalando que: "es preocupación preferente del Supremo Gobierno estimular la creación de instituciones particulares de enseñanza que integren el sistema educacional" (Extraído de Espinoza y González, 1993, p. 83).

Incluso en 1975, la Comisión Ortúzar ya discutía sobre la garantía constitucional de la Libertad de Enseñanza, que luego quedaría consagrada en la Ley Orgánica Constitucional de Enseñanza (LOCE). Sergio Diez, una voz autorizada al interior de la Comisión, apuntaba que era tarea del Estado cooperar con los privados en la labor escolar, no al revés: "Debe consagrarse en la Constitución el principio de que la sociedad debe colaborar al funcionamiento de la educación privada. No se trata solo de garantizar la libertad de enseñanza, por cuanto ello no es suficiente para un país como Chile" (Pérez-Navarro y Rojas-Murphy, 2017, p. 14).

Lo anterior da cuenta cómo desde los primeros años de la dictadura se propuso atender de manera preferente a las escuelas particulares por sobre las estatales, cuestión que sobrepasa las propuestas planteadas por Milton Friedman (1955), pues no se trataría solo de "echarlas a competir" sino derechamente de desmantelar el sistema público.

En cuanto al copago, llama la atención que en 1974 la Junta trató en más de una sesión la posibilidad de que las escuelas particulares subvencionadas cobren a las familias, sin con ello perder el aporte del Estado. El primer antecedente se encuentra a menos de cinco meses de la toma del poder. En sesión del 8 de febrero, el ministro de Educación, Castro Jiménez, presentó una fórmula de cálculo para incentivar a las escuelas particulares subvencionadas, y solicitó a la Junta autorización "para cobrar cierta cantidad a aquellos colegios gratuitos que se encuentren ubicados en barrios donde sea posible efectuar este cobro" (Acta $\mathrm{N}^{\circ} 88,1974$, pp. 2-3). Asimismo, en el contexto de la elaboración del presupuesto para 1975, la Dirección de Presupuestos (DIPRES) del Ministerio de Hacienda propuso que se incentive el copago de las familias en los establecimientos particulares subvencionados, arguyendo que quitar el financiamiento del Estado a las instituciones que cobren opera "en la dirección contraria de lo que queremos [...] Si nosotros le quitamos de la subvención lo que los colegios están recaudando por su propio esfuerzo, estamos quitándoles todo incentivo para que se haga" (Acta $\mathrm{N}^{\circ} 172 \mathrm{a}, 1974$, p. 11). Argumentos a favor del copago también se encuentran desde 1975 al interior de la Comisión Ortúzar (Pérez-Navarro y Rojas-Murphy, 2017).

En esta primera etapa también está presente el interés por financiar la educación vía voucher. En 1974, ODEPLAN propuso-sin éxito-implementar un voucher para administrar la alimentación a nivel escolar (Pérez-Navarro y Rojas-Murphy, 2017). En 1975, la misma ODEPLAN diagnosticó la falta de cobertura escolar en sectores marginales, proponiendo aumentar la injerencia del sector privado a través de un pago de subvención por matrículas desde el Estado (Espinoza y González, 1993), esto es, a través de un voucher.

De todos modos, esto facilitará la implementación posterior del modelo. El D668 de julio de 1974 fija el reglamento para los centros de padres y apoderados, dejándolos explícitamente fuera de cuestiones de orden pedagógico, administrativo y financiero, y normando su composición tutelada por el director del establecimiento y dos profesores designados por este. 
Todas estas propuestas se enmarcan en una situación de crisis financiera aguda, siendo el foco la disminución del gasto fiscal. En este contexto, en 1974 el ministro de Hacienda Jorge Cauas señaló a la Junta que "la casa [...] está medio quebrada" (Acta N¹60a, 1974, p. 36). En respuesta, Pinochet propone disminuir la jornada escolar a la mitad, para que el fisco ahorre en pagos de honorarios docentes, señalando que "los profesores pueden hacer clases particulares y aumentar, con ello, su renta" (Acta $\mathrm{N}^{\circ} 160 \mathrm{a}, 1974$, p. 11). Si bien estas medidas no prosperan, anuncian otro aspecto relevante dentro de la neoliberalización escolar: la precarización sistemática de las condiciones laborales de los profesores, entendiéndose como el principal elemento de ahorro para la caja fiscal.

Otro aspecto importante de esta etapa, son las propuestas de desconcentración administrativa y descentralización ${ }^{2}$ que retoman los esfuerzos de gobiernos anteriores y organismos internacionales para abordar el crecimiento de la matrícula que, hacía fines de los '60, volvió insostenible la centralización administrativa. Por tanto, esta idea nocentralista de la educación no es una cuestión original de la dictadura ni tampoco una cuestión puramente neoliberal ni exclusivamente chilena ${ }^{3}$ (Cox, 1985; Espinoza y González, 1993). Asimismo, la desconcentración administrativa no se restringía solo al ámbito educativo; era un objetivo declarado por el Régimen para promoverse en todo el aparato estatal. Como sostienen Espinoza y González (1993), en este período se mezclan desconcentración, descentralización y centralización: hay elementos de todas y ninguna se aplica en forma tan pura.

En 1975 ODEPLAN confecciona el Programa Ministerial de Educación que propone la descentralización del sector a través de organismos intermedios y un rol subsidiario del Estado, señalando:

Que el Ministerio de Educación deberá delegar progresivamente la gestión educativa, desestatizando el Sistema Educacional Estatal y descentralizándolo en lo administrativo y financiero, reservándose para sí la función de proveer los fondos necesarios para que los miembros de la sociedad accedan a la educación a que tienen derecho [...] La participación estatal en la matrícula debe ser reducida en 1975, proceso que deberá continuarse en 1976 (Espinoza y González, 1993, p. 75).

Este documento se adelanta en cinco años a las reformas de la década del 80, expresando nítidamente los objetivos políticos e ideológicos de la desconcentración administrativa (que sobreviene en municipalización), de un Estado financista, pero no proveedor del servicio escolar (que deviene en voucher), y de la privatización del sistema (con la consecuente desmejora de la educación pública).

La literatura diferencia entre desconcentración y descentralización. La desconcentración hace referencia a la delegación de determinadas funciones en organismos regionales o locales, pero que no son autónomos y dependen del gobierno central en términos presupuestarios y/o decisionales. La descentralización, en cambio, se define como la transferencia de atribuciones desde el nivel central a entidades locales, incluyendo poder de decisión y cierta autonomía (Espinoza y González, 1993).

3 Desde los '70 la Organización de Estados Americanos (OEA) y la UNESCO promovieron la descentralización y la desconcentración en América Latina (Espinoza y González, 1993). 


\section{SEGUNDA ETAPA (1976-1978): FORTALECIMIENTO DE ODEPLAN Y PRIMERAS PRIVATIZACIONES}

La segunda etapa es clave en varios aspectos. Se da continuidad y se profundiza en las tareas de desconcentración y descentralización. También se producen las primeras privatizaciones de establecimientos escolares y comienzan a delinearse las distintas posturas dentro del Régimen en relación con la orientación que debía tomar la política educativa: la «voz educativa» y protectora del Ministerio de Educación, versus la mirada privatizadora y pro mercado de la «voz económica», constituida por ODEPLAN y Hacienda (Cox, 1989).

Un primer aspecto por despejar tiene que ver con la influencia que habría ejercido Friedman sobre las políticas adoptadas por la dictadura en esta etapa. A juicio de PérezNavarro y Rojas-Murphy (2019), su visita en 1975 habría sido crucial para definir el corte neoliberal de las políticas educativas que comienzan a delinearse en 1976. No obstante, sin ánimo de minimizar la influencia de Friedman, no parecen haber evidencias concretas que apoyen esta afirmación. A modo de ejemplo, el Plan de recuperación económica implementado por la dictadura en 1975 (conocido como «terapia de shock»), ha sido erróneamente atribuido a los consejos de Friedman. Lo cierto es que dicho Plan fue aprobado por la Junta antes de su visita al país, y anunciado públicamente meses antes de la carta donde Friedman señala a Pinochet la necesidad de aplicar una «terapia de shock» (Montes, 2016). De hecho, las evidencias indican que en esta etapa más bien se continúan y profundizan las tareas adoptadas entre 1973 y 1975.

En efecto, en 1976 se inicia una etapa centrada en la profundización de la descentralización. En particular, la descentralización en el ámbito de la educación impulsada por ODEPLAN y la DIPRES, y no por Educación-, se lleva a cabo por dos vías estrechamente vinculadas, pero no equivalentes: privatización y municipalización (Espinoza y González, 1993).

La privatización comienza en 1977 en San Fernando, con el primer caso de traspaso, en particular, de una escuela agrícola de propiedad fiscal a la Corporación de Desarrollo Social de Educación Rural (CODESSER), creada un año antes por la Sociedad Nacional de Agricultura (SNA). CODESSER surge de conversaciones que sostuvo ODEPLAN con agrupaciones empresariales desde 1974, con el fin de estrechar el vínculo entre la Educación Media Técnico Profesional (EMTP) y el sector productivo (Espinoza y González, 1993). En términos jurídicos, se entregaron escuelas a corporaciones sin fines de lucro, pero constituidas por organizaciones empresariales lucrativas. Se trataba de una fórmula de «administración delegada»-apoyada por una Ley de noviembre de 1977 impulsada por ODEPLAN-, que permitía la enajenación de bienes del Estado y su administración en manos de particulares. En 1978 se transfirieron seis escuelas más a CODESSER.

Además de vincular de forma directa al sector productivo con los establecimientos de EMTP, la «administración delegada» buscaba instalar una administración de carácter empresarial en estos establecimientos (Espinoza y González, 1993). Se trataba de una política no solo anti-estatista, sino derechamente pro-empresarial. De hecho, estas normativas y traspasos son la antesala de la política del voucher oficializada durante los 80 , puesto que ya contemplaban una subvención no basal sino por promedio de asistencia.

La segunda vía de descentralización del sistema educativo es la municipalización, cuyo respaldo normativo fue promulgado en enero de 1976 a través de la Ley Orgánica de Municipalidades, articulando las visiones de ODEPLAN y DIPRES -en concreto de 
Miguel Kast y Juan Carlos Méndez, sus respectivos directores-. En la misma línea, en 1977 se dicta el Decreto Supremo (DS) 971 que aprueba la Estrategia Nacional de Desarrollo Económico y Social, elaborada por ODEPLAN, que propone traspasar progresivamente las escuelas fiscales a "organizaciones intermedias de la comunidad" (D(ex)114, 11/07/1983, p. 3). Todavía no estaba claro que esos organismos intermedios serían los municipios, pero ya en 1977 estaba delineada y normada jurídicamente la desestatización del sistema escolar.

En forma paralela a estas ideas privatizadoras y municipalizantes, y en evidente desconexión con ODEPLAN y la DIPRES, el Ministerio de Educación -que seguía manejado por militares- preparaba una Ley de Carrera Docente de carácter centralista y administrativa. Esto, da cuenta de la baja influencia que tiene en esta etapa la «voz educativa» en la definición de políticas para su sector.

Estas dos visiones encontradas respecto de la administración escolar y el modelo educativo en general, también se expresan al interior de la Junta de Gobierno. En una sesión de abril de 1978, relativa a la aprobación de la idea de legislar sobre el cobro de matrícula en liceos fiscales, se produce una discusión entre el Almirante Merino, quien estaba a favor, y el General Leigh, quien se mostraba más reticente. Aun cuando esta normativa no prospera, Merino seguirá defendiendo, en sesiones posteriores, el cobro de matrícula por los ingresos fiscales que dicho cobro traería (Acta $N^{\circ} 361 \mathrm{a}, 1978$ ), en línea con su defensa al proyecto de ley que buscaba aumentar la subvención a los establecimientos particulares subvencionados (Acta $\mathrm{N}^{\circ} 363 \mathrm{a}, 1978$ ).

La discusión sobre el proyecto de Carrera Docente que se venía trabajando desde Educación, entrega más evidencias de esta ambivalencia presente en los altos mandos de la dictadura. En sesión de septiembre de 1978, la discusión gira en torno a la fijación de salarios para los profesores de establecimientos subvencionados, cuya normativa (D456 de 1974) establecía un pago mínimo del $75 \%$ de la remuneración de los profesores fiscales que ejercían funciones similares. Al respecto, el ministro de Hacienda Sergio de Castro sostuvo que "cuando el Estado interviene en la fijación de precios, lo único que hace es echar a perder las cosas y establecer monopolios [...] Es obvio que la ley de salario mínimo lo que hace es contribuir a la alta tasa de desempleo" (Acta $\mathrm{N}^{\circ} 354 \mathrm{a}, 1978$, p. 8). Ante algunos cuestionamientos de la Junta, De Castro agregó que "es preferible que esa persona gane algo a que gane cero [...] Si el Estado se equivoca en la determinación de un precio [...] y lo coloca por encima de lo que sería un equilibrio natural, lo que está haciendo es que por ley está destinando a esa persona a la desocupación" (Acta N³54a, 1978, p. 8).

Merino parece convencerse con los argumentos de De Castro, proponiendo la eliminación de todas las normativas en relación con el pago de remuneraciones de profesores en educación particular -subvencionada y pagada-, de manera que se fijen "remuneraciones mínimas, lo más bajas posible, y nada más" (Acta N³54a, 1978, p. 9).

Finalmente, en línea con las propuestas precarizadoras y pro mercado de Merino y De Castro, la Junta acordó establecer un pago mínimo del 75\% para los profesores de escuelas particulares y subvencionadas, pero ya no en relación con los profesores del sector fiscal, sino del salario mínimo (Acta $\mathrm{N}^{\circ} 354 a$ ). En definitiva, se quitó toda protección salarial a los docentes no fiscales, anticipando la reforma de 1981 que extenderá esta precariedad y desprotección a casi todo el universo docente. Esto da cuenta del interés por dejar al mercado como el único regulador de las remuneraciones, al mismo tiempo que confirma el afán político de la dictadura por generar las condiciones normativas de desmejora, infravaloración y precarización del trabajo docente. 


\section{TERCERA ETAPA (1979): LA DICTADURA TOMA UNA DEFINICIÓN: DIRECTIVA PRESIDENCIAL Y PROYECTO DE MUNICIPALIZACIÓN}

En esta etapa se formulan dos documentos clave que orientarán las bases y que contienen los componentes del sistema educativo que funcionará al menos hasta $2015^{4}$. De ahí que el año 1979 constituya una etapa en sí misma, toda vez que las decisiones que se toman marcan un punto de inflexión en la configuración del sistema que cambió la educación escolar en Chile. La mayor parte de los análisis sobre el período ubican a la Directiva Presidencial en Educación como la única y gran directriz de la reforma escolar que comienza en 1981. Sin embargo, existió otro documento emanado desde la DIPRES que, si bien no alcanzó notoriedad en el debate público, igualmente se impuso y estableció los cimientos presupuestarios -de implicancia ideológica- que más tarde alcanzarán su promulgación jurídica. Desde nuestra perspectiva, este cariz subterráneo es fundamental para comprender las ideas de voucher y municipalización a través de la canalización de fondos de subvención vía municipios. De ahí que también sea fundamental en la comprensión del proceso.

La Directiva presidencial, junto a la carta que la acompañó y el discurso de Pinochet para inaugurar el año escolar de 1979, configuraron un todo coherente y orgánico en relación con las bases ideológicas del proceso de privatización del sistema educacional en Chile (Valdivia, 1979). Las etapas reseñadas hasta acá confirman que su contenido comprende algunos de los principales elementos que se venían discutiendo desde 1974.

El protagonismo alcanzado por la Directiva en los discursos, entrevistas y discusiones de la época responde a que se trató del primer documento público y de carácter ideológico en materia educativa de la dictadura (PIIE, 1991). La Directiva es la primera formulación concreta para comprender la transformación del modelo, en la medida que contiene tres de los componentes que proponemos como fundamentales en el proceso de neoliberalización del sistema escolar: privatización, copago y precarización laboral docente.

En términos de privatización, la Directiva confirma la intención política de favorecer la educación privada en desmedro de la pública. En la carta firmada por Pinochet que acompaña la Directiva se lee lo siguiente:

La posibilidad que el Estado expanda aún más su labor educacional debe considerarse improbable, atendidas la magnitud alcanzada por aquélla, la escasez de recursos, la urgencia de emplearlos en consolidar la obra ya realizada en este campo, y la existencia de otras necesidades sociales, también prioritarias. Por consiguiente, se estimulará con energía la ayuda que el sector privado presta a la tarea educacional (El Mercurio, 06/03/1979).

Este «todo coherente» constituido por la carta, el discurso y la Directiva, también explora nuevos mecanismos de apoyo a la participación privada en educación. Entre otras cosas, anuncia facilidades a través de créditos y subsidios para la inversión privada en construcción, arreglos y equipamiento escolar, considerando la incorporación del mercado inmobiliario en el sector. El flamante ministro de Educación, Gonzalo Vial, incluso anuncia una lógica de "comoditización" de los estudiantes, señalando que "estos créditos podrán cancelarse en «alumnos-años» de educación gratuita o semigratuita” (Qué pasa, 03/08/1979,

En 2015 se promulga la Ley de Inclusión que, a grandes rasgos, prohíbe el lucro, la selección y el copago en las escuelas y liceos que reciben subvención del Estado. A nuestro juicio, constituye la primera política escolar desde 1973 con una orientación claramente no neoliberal o que al menos pretende frenarla. 
p. 8). En otras palabras, sería un "canje": la atención gratuita de una determinada cantidad de estudiantes serviría para pagar la deuda.

Respecto del copago, y en clara relación con El Ladrillo -texto señero de las políticas neoliberales de la dictadura-, la Directiva establece una equivalencia entre participación social y pago de las familias, planteando que: "otra de las maneras de incentivar la participación comunitaria será la creación de un sistema a través del cual la comunidad organizada, a nivel local, apoye a los establecimientos escolares. En este sentido se aplicará en cada establecimiento un pago de derecho de escolaridad en la Enseñanza Media, que será voluntario" (El Mercurio, 04/04/1979, p. 7c).

En relación con la precarización laboral docente, la Directiva promueve una lógica educativa basada en mecanismos de premios y castigos que será crucial hasta la actualidad (Villalobos, 2016). Al respecto, señala que: "habrá premios para quienes se destaquen positivamente y penalidades indirectas para aquellos que no alcancen un nivel mínimo" (El Mercurio, 06/03/1979). Al mismo tiempo, la Directiva promovió que la formación de profesores rurales fuese sin requisitos y en menos años de estudio, impulsando la formación deficiente de quienes enseñarían, precisamente, en lugares con menores recursos y más necesidades.

Se trata de una etapa en que la figura de Vial fue protagonista y la «voz educativa» tomó fuerza. Esto es relevante por varias razones. La primera, porque Vial es el primer ministro de Educación no militar tras casi seis años de dictadura. La segunda, porque el peso de Vial suscitó que Pinochet (en tanto «voz política») otorgase mayor importancia a la «Voz educativa» que a la económica. Lo anterior es confirmado por algunos especialistas y actores clave del período ${ }^{5}$, también por el hecho que los contenidos de la Directiva no fueron discutidos en ninguna sesión de la Junta, lo que corrobora que ésta fue conceptualizada y escrita por Vial. En tercer lugar, la centralidad de Vial y la «voz educativa» rebate aquellos análisis que ponen en duda su rol en la privatización, toda vez que -sostienen- habría sido contrario a algunos de los principios que terminaron imponiéndose (Cox, 1985 y entrevista con C. Cox el 15/01/2018). Lo cierto es que Vial era un ferviente partidario de la privatización como muestran el contenido de la Directiva y sus declaraciones: "una meta a la que el Ministerio de Educación concede primordial importancia es la del fomento de la educación particular [...] que se tome conciencia del papel subsidiario que le corresponde al Estado en materia de educación" (Qué pasa, 18/10/1979: p. 37).

La publicación de la Directiva desencadenó el primer debate abierto sobre educación en seis años, posible de rastrear a través de los medios de prensa escrita. Como aproximación a las posturas partidarias, nos parece relevante citar el análisis de un columnista del semanario Qué Pasa:

En el $80 \%$ de los hogares de Santiago se gasta más en cigarrillos que en educación [...] ¿Por qué ocurre esto? La causa no es que la gente valore poco la educación [...] Lo que ocurre es simplemente que la educación es gratuita y, por lo tanto, no debe pagarse. La paga el Estado. Y como viene haciéndolo desde hace 100 años, a todos parece lógico que así sea. Por esto las familias chilenas destinan muy poco a educación, y por esto los recursos totales que existen para financiar la educación son insuficientes. Y por esto los profesores son mal pagados y el equipamiento de los establecimientos educacionales es deficiente. Y

Nos referimos especialmente al sociólogo y especialista en educación Cristián Cox (entrevistado el 15/01/2018) y a María Teresa Infante, clave en la implementación del nuevo sistema (entrevistada el 11/01/2018). 
por esto la educación en nuestro país no es excelente. Todo deriva del concepto artificial del Estado Docente, basado en ideologías del siglo XIX. Si en el pasado la ideología hubiera preconizado al "Estado Fumador" y a todos nos regalaran los cigarrillos y mientras tanto ese gasto lo destináramos a educación, hoy fumaríamos mal tabaco pero tendríamos una excelente educación (Qué Pasa, 18/10/1979, p. 41).

Por su parte, la mayor parte de las reacciones en contra de la Directiva, apuntaron a los sesgos ideológicos en materia de privatización y al poco presupuesto destinado al Ministerio, que demostraría la escasa importancia otorgada por la dictadura a la educación escolar y el interés manifiesto por dejar de concebir la educación como un derecho.

Ahora bien, si la Directiva anunciaba las ideas de privatización, copago y precarización laboral docente, el documento "Descentralización de la Gestión Educacional en Chile", elaborado por el Sector de Educación de la DIPRES del Ministerio de Hacienda en julio de 1979, formulaba las primeras propuestas en torno al voucher y la municipalización. Voucher y municipalización constituyen, entonces la cara menos visible de esta etapa. Desde la DIPRES "surgió, por primera vez durante el Régimen militar, una propuesta concreta de municipalizar la educación, como una opción diferente a la privatización de los establecimientos y complementaria a la idea de desconcentración administrativa del Ministerio de Educación" (Espinoza y González, 1993, p. 110). Dicha propuesta ha sido catalogada como «pragmatista», pues su origen emana de la dificultad de distribuir las subvenciones y de regular su adecuada utilización (Espinoza y González, 1993). Por tanto, su objetivo era abordar un problema técnico y no necesariamente alcanzar la institucionalización de determinadas bases ideológicas libremercadistas.

En términos generales, el documento de la DIPRES -coordinado por Patricio Arriagada, jefe delSectordeEducación delorganismo-planteabaun proyecto gradualdedescentralización para la educación a través de la municipalización, que iba en contra de las propuestas de regionalización que se venían implementando y que implicaban desconcentración más que descentralización (Espinoza y González, 1993). Juan Carlos Méndez -director de la DIPRES- fue clave en la configuración de esta idea de municipalización y en su posterior implementación (entrevistas a M.T Infante, 11/01/2018 y A. Prieto 23/01/2018).

Durante 1979, ODEPLAN se sumará a la DIPRES en el desarrollo y empuje de la propuesta de municipalizar la educación vía voucher. Para ello, ubica a María Teresa Infante, una de sus funcionarias de confianza, en la Superintendencia de Educación, puesto que "siempre que ODEPLAN deseaba impulsar alguna reforma, destinaba a alguien de su planta a los respectivos ministerios" (Entrevista a Patricia Matte en: Espinoza y González, 1993, p. 113)

Finalmente, entremedio de problemas entre Vial y Manuel Contreras -Director de la Central Nacional de Inteligencia $(\mathrm{CNI})^{6}-$, Pinochet le quita peso a la «voz educativa» en favor de la «voz económica», tomando la propuesta DIPRES-ODEPLAN e impulsando su implementación acelerada (como se verá en la siguiente etapa). La relación "virtuosa" entre DIPRES y ODEPLAN permitió llevar a cabo una reforma educativa radical, estructural y casi sin precedentes en el mundo.

Por último, el DL3063 promulgado el 29 de diciembre de 1979, hace posible el traspaso de escuelas y liceos fiscales a los municipios. Este Decreto Ley que no emana desde Educación sino desde Interior, se presenta bajo el eufemismo de Normas sobre Rentas

Entrevista a C. Cox, 15/01/2018. Para mayor información, ver Salazar (2013). 
Municipales y coincide con la salida de Vial y la entrada de Alfredo Prieto como ministro de Educación, quien se desempeñaba como subsecretario de la cartera desde 1976.

\section{CUARTA ETAPA (1980-1982): MUNICIPALIZACIÓN Y PRIVATIZACIÓN POR DECRETO}

Esta etapa está marcada por la ejecución de la gran reforma estructural al sistema escolar impulsada por la dictadura, expresada en la municipalización y privatización del $84 \%$ de las escuelas y liceos fiscales del país, y la instauración del voucher como mecanismo de financiamiento. Al mismo tiempo, se da un paso decisivo en la desconcentración del Ministerio al establecer que las funciones docentes pasan a formar parte de los municipios, pero no así los profesores, quienes además de perder su calidad de funcionarios del Estado, no serán considerados funcionarios de la planta municipal y se regirán "en todo por las normas laborales, de remuneraciones y de previsión aplicables al sector privado" (DFL1, 13/06/1980, p. 1). En definitiva, en esta etapa se comienzan a implementar decididamente cuatro de los cinco elementos que identificamos como característicos del proceso de neoliberalización de la educación chilena: municipalización, privatización, voucher y precarización docente.

En cuanto a la municipalización, el ministro Prieto corrobora que la idea nace desde el Ministerio de Hacienda y no desde Educación, al expresar que "la Ley de Carrera Docente fue el factor que más apuró la municipalización [...] era una bomba de tiempo por cuanto suponía un financiamiento adicional muy fuerte y un problema de burocratización [...] La municipalización fue una medida que tuvo que adoptarse con urgencia" (Espinoza y González, 1993, p. 149). Al respecto, en diciembre de 1981, la Junta deroga los artículos relativos a la Carrera Docente que venía impulsando la cartera de Educación desde 1977, puesto que, producto de la municipalización, los profesores dejarían de ser funcionarios públicos (Acta $\mathrm{N}^{\circ}$ 41/81, 1981).

En síntesis, la decisión medular de municipalizar la educación no la toma Prieto, pues no se sanciona en el Ministerio de Educación, sino en Hacienda y ODEPLAN. Lo que Educación hace, es decidir los tiempos y modos de ejecución, pero no el contenido de la política. Cuando Vial era ministro, la «voz educativa» era alta y la «económica» baja, pero con Prieto esto se invierte. No se trata de las características personales de Prieto $-\mathrm{o}$ cualquier ministro/a-, sino de que la coyuntura es otra: es la «voz política»-de Pinochetla que decide dar más poder a la «voz económica».

En junio de 1980, se promulga desde Interior el DFL1, que reglamenta el traspaso de los servicios educativos a los municipios, equiparando en la práctica a las escuelas municipales con las particulares subvencionadas. El 31 de diciembre de 1980, se realizó la primera ola de traspasos en diecinueve comunas: incluyó a 362 establecimientos, 3.097 profesores y 67.208 estudiantes (Espinoza y González, 1993). El proceso fue fulminante: en abril de 1982 se había traspasado el 84\% de los establecimientos fiscales, "5.724 planteles con 72.531 docentes y administrativos, con aproximadamente dos millones de alumnos" 7 (Espinoza y González, 1993, p. 120).

Ante la consulta sobre la celeridad del proceso, Alfredo Prieto señaló: "No tuve vacaciones. Yo me propuse: los alumnos se fueron de vacaciones en diciembre y en marzo ninguno entra a la institución de la cual se fue, entran todos a otra. Y los profesores entran en marzo y tienen otro empleador" (entrevista a A. Prieto, 23/01/2018). 
Aun cuando municipalización y privatización son procesos distintos, están estrechamente vinculados (Espínola, 1989; Espinoza y González, 1993). A juicio de Espinoza y González, "la municipalización era vista por las autoridades del Gobierno como una etapa intermedia a la privatización del sistema" (1993, p. 115). Esto se expresaría en el DL3476 dictado desde Hacienda en septiembre de 1980, que norma el traspaso de establecimientos a privados que no persigan fines de lucro, incluyendo la posibilidad que los municipios privaticen las escuelas que recibieron del Estado. De hecho, Carlos Cáceres, ministro de Hacienda en 1984, confirmó en pleno proceso que "el objetivo real sigue siendo la privatización [...] la municipalización es una etapa, es un paso" (Cox, 1985, p. 163).

El voucher, por su parte, se establece a través del mismo DL3476. Este decreto instituye las normas para el pago de la subvención, estableciendo un «valor unitario por alumno» que se pagaría mensualmente (y no anualmente como antes), reajustable con la UTM y en función de la asistencia media de los estudiantes. Este decreto permite que los liceos municipales puedan hacer un cobro mensual (derecho de escolaridad), sin limitación de un monto máximo, pero de carácter voluntario. También permite un cobro de matrícula anual, que establece un máximo. Este punto generó grandes discusiones al interior de la Junta. Pinochet e incluso Merino (que en 1978 se mostró a favor), se negaron a que la educación fiscal implicara un cobro, pero como normativamente las escuelas traspasadas se homologaban a las particulares subvencionadas, se abría la puerta para que también pudieran hacerlo. De Castro, desde Hacienda, defendía la posibilidad de cobro (Acta $\left.\mathrm{N}^{\circ} 404 \mathrm{a}, 16 / 12 / 1980\right)$.

Lo particular del voucher son tres cuestiones inéditas en la historia educativa del país: 1) se vuelve el único mecanismo de financiamiento, homogeneizando escuelas privadas y públicas (Slachevsky, 2015); 2) entrega un enorme volumen de recursos -sin precedentesdesde el Estado a las escuelas privadas (Espínola, 1989); 3) no solo cambia el mecanismo de asignación de recursos, sino también el uso de estos. En otras palabras, permitió que las escuelas privadas dispusieran casi libremente de los recursos, generando mecanismos para aumentar la rentabilidad en desmedro del salario del profesorado. A modo de ejemplo, durante la década del 80 las escuelas municipales destinaban casi el $90 \%$ de sus recursos a sueldos, mientras que las particulares subvencionadas entre un $40 \%$ y un 50\% (Espínola, 1989). A juicio de Espínola (1989), este cambio en la asignación de recursos vía voucher, sería el más importante de todos los introducidos por la dictadura, puesto que sobre este descansaría el resto de las modificaciones.

Además de desmembrar el sistema público y centralizado, el voucher buscaba declaradamente incentivar al sector privado a abrir nuevas escuelas dado su atractivo como negocio (Espínola, 1989). Y logró su cometido. El número de planteles privados subvencionados aumentó en un 57.9\% entre 1980 y 1985; crecimiento que no se explica por un aumento de la matrícula, que apenas varió un 4\% (Espinoza y González, 1993). Más bien hubo una redistribución de los estudiantes dentro del sistema. Entre 1980 y 1989, la matrícula de los particulares subvencionados aumentó de un $14 \%$ a un $31 \%$, mientras que la matrícula municipal/fiscal bajó del 79\% al 61\% (Espinoza y González, 1993).

Por último, nos parece destacable que, en sesión de abril de 1980, el ministro Prieto solicitara facultades a la Junta para exonerar profesores opositores al Régimen. Sin dudarlo, la Junta autoriza al recién asumido ministro para hacerse cargo de una lista entregada por la CNI, "por región y por escuela que sobrepasa los 3 mil a 4 mil profesores. Esa gente no la podemos tener dentro del sistema" (Acta $N^{\circ} 392 \mathrm{a}, 1980$, p. 53). 
A mediados de 1982, la economía comenzó a contraerse aceleradamente, iniciando una aguda crisis financiera que originó la desindexación de la subvención de la UTM. Esto generó desincentivos en el sector privado y déficits en los establecimientos municipales (Espinoza y González, 1993), y derivó en el primer congelamiento de traspasos por falta de recursos ${ }^{8}$. Prieto sale del Ministerio en abril de 1982 y en su reemplazo asume Rigoberto Cruz Johnson, un militar que duró cuatro meses en el cargo, inaugurando una etapa compleja y llena de dudas para el Régimen.

\section{QUINTA ETAPA (1983-1985): CRISIS DE LA DIMENSIÓN FUNDACIONAL}

La quinta etapa está marcada por la profunda crisis económica iniciada en 1982 que significó la salida de varios Chicago Boys de los altos mandos. Esta fuerte crisis implicó que la dictadura tambaleara, modificara su agenda y torciera el rumbo, para enfocarse en solucionar urgencias coyunturales (PIIE, 1991). Manuel Antonio Garretón (1983) llamó a esta etapa la «crisis de la dimensión fundacional». En el sector escolar, se caracterizó por el sucesivo congelamiento de los traspasos, una alta rotación de ministros y un recorte al monto de la subvención transversalmente criticado. En términos ideológicos, dentro de las filas del Régimen, se levantaron voces que pusieron en tela de juicio los pilares del sistema implantado. Al mismo tiempo, otros sectores oficialistas realizaron una defensa cerrada del modelo, que progresivamente fue en ascenso en la medida que los índices económicos fueron mejorando.

En febrero de 1983, tras dos ministros que duraron pocos meses, la abogada Mónica Madariaga asumió una cartera de Educación en crisis. La recién asumida ministra si bien era contraria al Estado Docente, comenzó a cuestionar los principios del modelo: "Tanto el Estado como el sector privado tienen un campo propio de acción y ni uno ni otro deben salirse de él [...] No se debe caer en la posición de estatizarlo todo, así como tampoco en aquella que postula privatizarlo todo" (La Nación, 29/06/1983, p. 3). Esto derivó en la suspensión definitiva de los traspasos. Para Madariaga, la suspensión no era transitoria, pues consideraba "conveniente que el Estado mantenga su tuición absoluta, aunque sea sobre un modesto 16\% de los establecimientos educacionales del país" (La Segunda, 10/10/1983).

La suspensión de los traspasos en 1983 emana de la «voz educativa» en un contexto de resistencias del gremio docente y del mundo civil. No obstante, tras ocho meses de polémica gestión, la «voz política» decide reducir la autoridad de la «voz educativa»y reemplazar a la ministra Madariaga por Horacio Aránguiz Donoso, historiador y dueño de colegios privados. Si bien con este cambio se retoman moderadamente los traspasos, en junio de 1984 se vuelven a paralizar.

En este período crítico, el profesorado continuó siendo el actor más perjudicado. Al no estar vinculada con la UTM, la subvención no se reajustó de acuerdo con la inflación, lo que en términos reales implicó una disminución drástica de sus sueldos. Dada la libertad casi total que tenían los establecimientos particulares subvencionados para hacer uso de los recursos, pagaban en promedio la mitad que a un profesor municipal en las mismas funciones. "Los datos [...] muestran cómo el máximo peso de la tensión económica recae sobre los profesores" (Espínola, 1989, p. 62).

Durante el período 1982-1986 se congelaron varias veces los traspasos, aunque solo el anuncio de septiembre de 1983 puede considerarse un congelamiento "definitivo", pues en todos los otros casos el congelamiento es nominal, pues se continúa con los traspasos, aunque en cantidades muy menores. 
El 29 de julio de 1985, Sergio Gaete es designado ministro de Educación. Ese mismo año, se avizoran los primeros signos de recuperación económica, reapareciendo con fuerza la defensa a los principios del modelo en medios de prensa como El Mercurio, La Segunda y La Nación. Se renueva así, al interior de la dictadura, la convicción de continuar con los traspasos.

\section{SEXTA ETAPA (1986): REARTICULACIÓN IDEOLÓGICA Y REANUDACIÓN DE TRASPASOS}

El año 1986 también es una etapa en sí misma. Se reanudan y completan los traspasos del $16 \%$ de escuelas que faltaban ${ }^{9}$, con fuertes manifestaciones de rechazo. Asimismo, la rearticulación ideológica dentro del Régimen vuelve a poner en primera línea las ideas neoliberales, al tiempo que Hernán Büchi, desde el Ministerio de Hacienda, comienza a capitalizar políticamente la recuperación económica. Esta sexta etapa funciona como bisagra entre un período de crisis y otro de auge económico y político. En lo escolar, culmina el proceso de descentralización vía municipios con la cimentación de un modelo neoliberal que perdura casi inalterado hasta 2015.

El 26 de mayo de 1986, el ministro Gaete anunció a través de los medios que en agosto se retomarían y culminarían los traspasos (La Segunda, 27/05/1986). Este anuncio redundó en la más grande ola de protestas, paralizaciones y críticas del sector educativo durante toda la dictadura en la que participaron secundarios, universitarios y profesores. Sin embargo, como desde el oficialismo ya se había superado la etapa dubitativa -respaldada por el crecimiento económico-, se desplegó una defensa comunicacional con la misma fuerza, sustentada en el virtuosismo de la competencia, la eficiencia de los privados, el antiestatismo y la municipalización (El Mercurio, 29/05/1986).

La municipalización también comenzó a defenderse bajo el argumento del mayor control que permitía sobre los docentes. Lucía Hiriart, esposa de Pinochet, señaló abiertamente que cuando los profesores dependen de un municipio, "están más cercanos a un lugar donde se les puede controlar; donde se puede saber si cumplen con las horas de clases; si llevan a cabo programas de educación y no programas políticos" (Revista Análisis, 10/06/1986, pp. 13-14).

Mientras tanto, el Ministerio de Educación intentaba sacar adelante el proyecto de Estatuto Docente para las escuelas municipales y particulares subvencionadas sin fines de lucro. En dos sesiones de la Junta sobre el tema, Büchi se opone al proyecto por el alto costo que implicaría para el fisco, y porque -a su juicio- sería una intromisión del Estado en cuestiones que podrían manejarse por la Ley de oferta y demanda (Acta $\mathrm{N}^{\circ} 35$, 1986). Asimismo, en mayo de 1986, se promulga el $\mathrm{D}(\mathrm{ex}) 296$, que permite el cobro de matrícula en liceos fiscales, en circunstancias que la Junta -en especial Pinochet- había rechazado anteriormente en dos oportunidades esta posibilidad. La «voz educativa» y la «Voz económica» volvían a enfrentarse, y la «Voz política» nuevamente entregaba mayor poder a la segunda. De hecho, la decisión de retomar los traspasos "surgió originalmente

En estricto rigor, dado que durante 1983 y 1984 se realizaron algunos traspasos -muy pocos-, para 1986 esta cifra bordeaba el 14\%. No obstante, optamos por mantener la cifra de $16 \%$, dado que facilita la línea argumental sin afectar la explicación de los procesos. 
de la Secretaría de Desarrollo y Asistencia Social (dependiente de la Presidencia de la República) en concordancia con ODEPLAN y sin la aquiescencia previa del Ministerio de Educación” (Espinoza y González, 1993, p. 134). Una clara expresión del conflicto entre estas «voces» se observa en la salida "voluntaria" de Gaete del Ministerio en julio de 1987, la que se produce tras la decisión de Büchi de despedir a más de ocho mil profesores mientras Gaete estaba de vacaciones (La Época, 28/06/1987).

Así culmina la puesta en marcha de una de las reformas escolares de mayor envergadura e impacto del siglo XX en Chile, cuyo punto neurálgico -tal como propone Friedmanradica en que el Estado deja de proveer el servicio educacional. No obstante, esto no significará su retirada, por el contrario, el Estado se convierte en el encargado de entregar financiamiento al $93 \%$ de la matrícula escolar vía municipios y escuelas particulares subvencionadas, bajo la lógica del voucher igualitario. En efecto, el Estado cumple un rol fundamental y activo en la neoliberalización del sistema escolar chileno, toda vez que crea un mercado de educación escolar donde antes no existía. Bajo la variante del neoliberalismo emulador (Maillet, 2015), el Estado, además de promover y posibilitar el funcionamiento de las reglas del mercado, organiza las condiciones necesarias para que la competencia pueda operar dentro de un sistema que, histórica y comparativamente, nunca antes había funcionado de esa manera (Bellei, 2015; Vergara y Martin, 2017).

\section{SÉPTIMA ETAPA (1987-1990): LA CONSOLIDACIÓN DE LAS BASES DEL MODELO}

La séptima y última etapa del esquema de organización que proponemos se caracteriza por la consolidación de las bases del modelo neoliberal en educación escolar. En esta etapa la «Voz económica» se vuelve hegemónica, apoyada en tasas de crecimiento del PIB que alcanzaron el 10\% en 1989 (Ffrench-Davis, 2003). Además, se organiza y ejecuta una política sistemática de despido de profesores, y se dicta una nueva ley de subvenciones que considera por primera vez el copago. Finalmente, se elabora, discute y aprueba la Ley Orgánica Constitucional de Enseñanza (LOCE) -mandato de la Constitución de 1980-, y se confecciona y pone en práctica el Sistema de Medición de la Calidad de la Educación (SIMCE).

Entre fines de 1986 y principios de 1987, los temores de los profesores se vuelven realidad. Hacienda comienza a presionar para disminuir el número de docentes en el sistema municipal y se reitera lo de la etapa anterior: mientras Educación pretendía aprobar el Estatuto Docente, Büchi desde Hacienda abogaba por la disminución de costos. En este contexto de disputa, incluso Merino, quien se mostró siempre proclive a la privatización y desmejora de las condiciones docentes, "defendió" a los profesores señalando que "por no pagar a los profesores lo que corresponde tenemos un país de enanos mentales"10 (Acta N44/86, 1987, p. 56). Sin embargo, en esta etapa la «voz económica» dominará por sobre la política, aprobándose un Plan de Racionalización de la administración educacional para ser implementado por la Subsecretaría de Desarrollo Regional y Administrativa con el objetivo de organizar los despidos masivos (El Mercurio, 04/02/1978, p. 4c).

10 Hacia el final del Régimen, las posiciones de Merino y Büchi se consolidan como antagónicas -dentro de acuerdos generales mayores-, al menos en temas educativos. 
Por otra parte, uno de los aspectos clave de esta etapa es la discusión y posterior aprobación de la LOCE, que fue extensamente debatida en seis sesiones de la Junta entre junio de 1987 y marzo de 1990. Si bien la LOCE se aprueba solo un día antes de que Pinochet entregue el mando político (10 de marzo de 1990), está lejos de responder a la improvisación. Por el contrario, la LOCE consagra un anhelo muy importante para la dictadura y largamente debatido desde la Comisión Ortúzar -establecida en 1973- en adelante, relacionado con la libertad de enseñanza, la que se consagra como libertad de emprendimiento; esto es, la libertad para que privados puedan abrir escuelas y recibir subsidio público por ello, sin mayores controles ni trabas.

La LOCE también incluyó como materia importante el SIMCE. Este sistema de medición que se instala en 1988 -aunque tiene antecedentes al menos desde 1983-, permite centrar el discurso en la calidad del sistema. Se sigue la propuesta de Friedman (1955) respecto a que un modelo de información de resultados académicos favorecería la competencia interna del sistema y permitiría la mejora constante, o bien la desaparición de las escuelas que no logren los mínimos. Serían los padres y madres quienes, «votando con los pies» (Atria, 2012), elegirían el mejor establecimiento para sus hijos, reforzando a su vez la supuesta lógica participativa del voucher, y favoreciendo la no-distinción de escuelas públicas y particulares subvencionadas. Este cambio se expresa simbólicamente en los textos legales emitidos entre 1987 y 1988, que "ya no distinguen entre administración municipal y administración particular, sino que se refieren en términos genéricos a la «educación particular gratuita»" (Espínola, 1989, p. 46).

En cuanto al copago, a fines de 1988, una vez que la dictadura ya había perdido el plebiscito, se promulga una normativa que permite y regula el financiamiento compartido en educación básica y media (Ley $\mathrm{N}^{\circ} 18.768$, del 29/12/1988); cuestión anhelada por algunos grupos desde los inicios de la dictadura. Como señala Espínola (1989), esto representa otro importante paso hacia la privatización del sistema escolar, porque al tiempo que disminuye todavía más la responsabilidad del Estado, se regula por ley el aumento de las diferencias entre escuelas de más y menos recursos. A esto habría que agregar que es también un paso decisivo hacia la mercantilización del sistema (Bellei, 2015), toda vez que favorece el uso del voucher desde una lógica individualizada, donde cada familia se autoclasifica en un segmento de "mercado escolar" (Jofré, 1988), favoreciendo la segregación en función de su capacidad de pago.

El fin de esta etapa es el fin de la dictadura. En este final se consolida el triunfo de la «voz económica» y se cimientan bases sólidas para la hegemonía del modelo, no solo porque se crea un mercado escolar, sino porque el modelo educativo permite estructurar la sociedad bajo esa lógica. En otras palabras, la importancia de la operatoria económica por sobre la lógica educativa tiene consecuencias hegemónicas sobre la sociedad y el sentido común. El modelo educativo impuesto por la dictadura obliga a la sociedad a operar bajo la lógica del pago, el voucher y la competencia, principios propios del mercado y contrarios a concebir la educación como un derecho.

\section{CONCLUSIONES}

El recorrido por estas siete etapas permite vislumbrar que la neoliberalización del sistema escolar fue un proceso que, si bien contaba con antecedentes políticos, económicos e ideológicos importantes, se comprende mejor como una senda que se va gestando y 
construyendo sobre la marcha. Cuyas definiciones responden siempre a contextos locales que van exigiendo una constante revisión y discusión, además de disputas de posiciones políticas que van mutando. En consecuencia, su implementación no responde a la imposición de una pauta prefijada, sino a una constante resignificación de ideas para la creación de un sistema que no contaba con ninguna referencia similar en el mundo.

Como indican Pérez-Navarro y Rojas-Murphy:

El discurso neoliberal radical que terminó por imponerse no siempre fue aceptado unánimemente por sus integrantes, incluso, existían en algunos miembros aprensiones al hecho de desligar al Estado de su rol regulador y coordinador en educación, lo que devela que el paso ulterior de un discurso a otro no estuvo exento de vicisitudes que el grupo economicista neoliberal tuvo que soslayar (2017, p. 21).

El recorrido por etapas propuesto permite comprender que desde la «voz política» -tributaria en gran medida del mundo castrense-, las modificaciones al sistema escolar estuvieron siempre sustentadas por una ideología de corte antiestatal y antimarxista, cuyos ejes fueron la precarización docente, la municipalización como «desconcentración centralizada» y el debilitamiento de la educación pública vía privatización. En tanto, para la «Voz económica», representada por el Ministerio de Hacienda y ODEPLAN, la implantación de un sistema escolar con lógica de mercado respondía a cuestiones presupuestarias -aunque inseparables del componente ideológico que las sustenta-, vinculadas con un financiamiento fiscal menor y descentralizado mediante el desarrollo de mecanismos como el voucher y el copago. Finalmente, el resultado es doble: se oblitera la «voz educativa»y, al mismo tiempo, se reorienta el Estado, pasando de ser el mayor proveedor y financista de educación escolar, a ser el organizador y financista de un mercado educativo.

Asimismo, el recorrido por estas siete etapas nos permite comprender que el proceso, en todas sus dimensiones, se relaciona con una ideología política que buscó y logró permear el sentido común, imponiendo un sistema a través del cual las mismas familias se autoclasifican por capacidad de pago (Jofré, 1988), y terminan reproduciendo un sistema individualizante, competitivo y segregador.

En consecuencia, este proyecto obedece a una nueva regulación política más que a una mera desregulación económica (PIIE, 1991). Durante estas siete etapas lo que se asegura son las bases de este proyecto político, pero no el modelo mismo. La operación completa y entrada en régimen del modelo de educación escolar neoliberal se produce en los 90 y su consolidación tiene en el informe Brunner de 1994 uno de sus hitos más relevantes. Comprender cabalmente el proceso de instauración del modelo neoliberal en educación escolar permite, no solo entender mejor aspectos clave del funcionamiento de este modelo en la actualidad, sino también, esperamos, abrir posibilidades para su transformación.

\section{REFERENCIAS BIBLIOGRÁFICAS}

Atria, F. (2012). La mala educación. Ideas que inspiran al movimiento estudiantil de Chile. Santiago: Catalonia.

Bellei, C. (2015). El gran experimento. Mercado y privatización de la educación chilena. Santiago: LOM Ediciones. 
Estudios Pedagógicos XLVI Nº 2: 321-340, 2020

HACIENDO NEOLIBERALISMO SOBRE LA MARCHA. CREACIÓN Y RESIGNIFICACIÓN DE IDEAS EDUCATIVAS

EN LA DICTADURA CHILENA

Brunner, J. J. (1982). Tendencias de cambio en el sistema de educación superior. Chile 1973-1982. Documentos de trabajo programa FLACSO $\mathrm{N}^{\circ} 152$. Santiago.

Castro-Paredes, M. (2012). Descentralización educacional en Chile: itinerario sin territorio, INNOVAR. Revista de Ciencias Administrativas y Sociales, 22(43), 77-92.

Cox, C. (1985). Hacia la elaboración de consensos en política educacional: actas de una discusión. Santiago: CIDE.

Cox, C. (1989). Sistema político y educación en los '80: medidas propuestas y silencios. En: GarcíaHuidobro, J.E. (editor) Escuela, calidad e igualdad: los desafíos para educar en democracia. Santiago: CIDE.

Espínola, V. (1989). Los resultados del modelo económico de la enseñanza básica: la demanda tiene la palabra. En: García-Huidobro, J.E. (editor) Escuela, calidad e igualdad: los desafíos para educar en democracia. Santiago: CIDE.

Espinoza, O. y González, L. E. (1993). La experiencia del proceso de desconcentración y descentralización educacional en Chile 1974-1989. Santiago: PIIE, Documento de trabajo.

Falabella, A. (2015). El mercado escolar en Chile y el surgimiento de la Nueva Gestión Pública: el tejido de la política entre la dictadura neoliberal y los gobiernos de la centroizquierda (1979 a 2009). Educação \& Sociedade, 36(132), 699-722.

Ffrench-Davis, R. (2003). Chile, entre el neoliberalismo y el crecimiento con equidad. Nueva Sociedad, (183), 70-90.

Friedman, M. (1955). The role of Goverment in Education. En: Robert A. Solo (Ed.) Economics and the Public Interest. New Brunswick, NJ: Rutgers University Press.

Garretón, M. A. (1983). El proceso político chileno. Santiago: FLACSO.

González, F. (2015). Mil días de la Junta Militar de Gobierno. La metamorfosis subterránea de la educación chilena durante los primeros años de la dictadura militar (1973 - 1979). Cuadernos Chilenos de Historia de la Educación, (4), 34-61.

Jofré, G. (1988). El sistema de subvenciones es educación: la experiencia chilena. Revista Estudios Públicos, (32), 193-237.

Maillet, A. (2015). Variedades de neoliberalismo. Innovación conceptual para el análisis del rol del Estado en los mercados. Revista de Estudios Políticos, (169), 109-136.

Montes, L. (2016). Milton Friedman y sus visitas a Chile. Revista Estudios Públicos, (141), 121171.

Pérez-Navarro C. y Rojas-Murphy, A. (2017). Estado Docente, subsidiariedad y libertad de enseñanza. El proceso de privatización educacional en Chile desde la perspectiva de los actores (1973-1990). Série-Estudos, Campo Grande 22(45), 5-23.

Pérez-Navarro, C. y Rojas-Murphy, A. (2019). Introducción y consolidación de los principios de mercado en el discurso oficial sobre educación de la dictadura civil militar chilena (19731990). Paulo Freire. Revista De Pedagogía Crítica, (20), 105-122.

PIIE (1991). Las Transformaciones de la Educación bajo el Régimen Militar, 1 y 2. Santiago: Programa Interdisciplinario de Investigaciones en Educación.

Salazar, M. (2013). Las letras del horror, Tomo II: la CNI. Santiago: LOM Ediciones.

Slachevsky, N. (2015). Una revolución neoliberal: la política educacional en Chile desde la dictadura militar. Educação e Pesquisa 41, 1473-1486.

Valdivia, T. (1979). El proyecto educacional del gobierno: interpretación en el contexto. Revista Mensaje, (278), 218-225.

Vergara, J. y Martin, A. (2017). Pensar la educación. Desde Friedman a Dewey. Santiago: Editorial Universitaria.

Villalobos, C. (2016). El campo educativo en Chile post-dictadura (1990-2013). Continuidad y ruptura en la implementación del neoliberalismo en educación. En: VV.AA. Democracia versus neoliberalismo: 25 años de neoliberalismo en Chile. Santiago: Fundación Rosa Luxemburgo, ICAL, CLACSO. 
Actas de la Junta de Gobierno

Acta $\mathrm{N}^{\circ} 3,1973$

Acta $\mathrm{N}^{\circ} 64,1974$

Acta $N^{\circ} 88,1974$

Acta $N^{\circ} 160 a, 1974$

Acta $N^{\circ} 172 \mathrm{a}, 1974$

Acta $N^{\circ} 354 a, 1978$

Acta $N^{\circ} 361 \mathrm{a}, 1978$

Acta $N^{\circ} 363 a, 1978$

Acta $N^{\circ} 392 \mathrm{a}, 1980$

Acta $N^{\circ} 404 a, 1980$

Acta $\mathrm{N}^{\circ} 41 / 81,1981$

Acta $\mathrm{N}^{\circ} 35,1986$

Acta $\mathrm{N}^{\circ} 44 / 86,1987$

Periódicos y revistas

El Mercurio, 04/02/1978

El Mercurio, 06/03/1979

El Mercurio, 04/04/1979

Qué pasa, 03/08/1979

Qué pasa, 18/10/1979

La Nación, 29/06/1983

La Segunda, 10/10/1983

La Segunda, 27/05/1986

El Mercurio, 29/05/1986

Revista Análisis, 10/06/1986

La Época, 28/06/1987 
Estudios Pedagógicos XLVI Nº 2: 321-340, 2020

HACIENDO NEOLIBERALISMO SOBRE LA MARCHA. CREACIÓN Y RESIGNIFICACIÓN DE IDEAS EDUCATIVAS EN LA DICTADURA CHILENA

\section{Entrevistas}

Cox, Cristián. Entrevista realizada en Santiago el 15/01/2018

Infante, María Teresa. Entrevistada realizada en Santiago el 11/01/2018

Prieto, Alfredo. Entrevista realizada en Santiago el 23/01/2018 